\title{
Synthesis of High Molecular Weight Nylon 6 by Anionic Polymerization of $\varepsilon$-Caprolactam. Formation of Cyclic Oligomers
}

\author{
Kazue Ueda, Masahiro Hosoda, Tsunetoshi Matsuda, and Kazuo TaI \\ Research \& Development Center, UNITIKA LTD., \\ 23 Kozakura, Uji, Kyoto 611, Japan
}

(Received June 6, 1997)

\begin{abstract}
The formation of cyclic oligomers in the process of the anionic polymerization of $\varepsilon$-caprolactam was studied. Cyclic oligomer contents increased with chain initiator concentration and decrease in catalyst concentration. Cyclic dimer content was much higher than that of cyclic trimer to cyclic hexamer and sensitively influenced by the concentrations of catalyst and chain initiator in comparison with cyclic trimer to cyclic hexamer. A mechanism is proposed in which cyclic dimer is peculiarly formed at the acyl-lactam terminal group.

KEY WORDS Cyclic Oligomer/ Cyclic Dimer/ Nylon 6/ Anionic Polymerization/ $\varepsilon$-Caprolactam/ Catalyst/ Chain Initiator /
\end{abstract}

The anionic polymerization of $\varepsilon$-caprolactam has been extensively investigated because of the high yield and rate of polymerization. ${ }^{1-4}$ Fundamental features such as various catalysts, ${ }^{2,5,6}$ propagation mechanism, ${ }^{7-10}$ and kinetics ${ }^{11-16}$ are well known.

The authors carried out systematic studies directed on high molecular weight nylon 6 and obtained stable high-molecular weight polymer (the weight average molecular weight, $M_{w}=9.5 \times 10^{5}$ ) by controlling the water content in monomer and the concentration of the catalyst and chain initiator. ${ }^{17-19}$ The kinetic equations of their anionic polymerization are in agreement with the ideal rate equation. ${ }^{20}$

The formation of cyclic oligomers in anionic polymerization of $\varepsilon$-caprolactam has not been studied in detail. Cyclic oligomer content (up to heptamer) has been measured only in a few studies. ${ }^{21-23}$ The proposed mechanism of cyclic oligomer formation still seems insufficient. ${ }^{24}$ This study deals with kinetic data of the cyclic oligomer formation in the polymerization as a function of the concentrations of catalyst and chain initiator. The mechanism of the formation is discussed.

\section{EXPERIMENTAL}

\section{Materials}

Industrial fiber grade $\varepsilon$-caprolactam as monomer, ethyl magnesium bromide (EtMgBr; Aldrich Chemical Co., Inc.) as catalyst in $1.0 \mathrm{moll}^{-1}$ tetrahydrofuran solution and $N$-acetyl- $\varepsilon$-caprolactam (Ac-CL; Tokyo Chemical Industry Co., Ltd.) as monofunctional chain initiator were used for polymerization.

\section{Polymerization}

Water in $\varepsilon$-caprolactam was removed to less than 0.013 mol\% by bubbling dry argon gas for $5 \mathrm{~h}$ in a three-neck flask at $120^{\circ} \mathrm{C}$. Water content was controlled by adding water to this $\varepsilon$-caprolactam and measured by a Karl Fischer auto titration equipment (Hiranuma industrial Co., AQV-5S). The chain initiator was injected into $\varepsilon-$ caprolactam with controlled water content. The mixture of monomer and initiator was poured into a glass tube (inside diameter $20 \mathrm{~mm}$, volume $20 \mathrm{ml}$ ) dried beforehand by heating above $100^{\circ} \mathrm{C}$ under $10 \mathrm{~Pa}$ vacuum. The catalyst $(\mathrm{Et} \mathrm{MgBr})$ solution was injected into the tube via a syringe, and the tube was sealed after unnecessary solvent was evaporated at $10 \mathrm{~Pa}$. The polymerization was carried out with shaking in an oil bath at $150^{\circ} \mathrm{C}$ (thermostated at $\pm 1.0^{\circ} \mathrm{C}$ ).

Polymerization was stopped by quenching to dry ice/methanol temperature $\left(-73^{\circ} \mathrm{C}\right)$. The product was shaved with a boring machine and analyzed.

\section{Analysis of Cyclic Oligomers}

Monomer and cyclic oligomers in the polymer were extracted by boiling water in a sealed tube for $5 \mathrm{~h}$. The monomer and cyclic oligomer contents in the extract were measured by liquid chromatography (HPLC, Column; Waters C18 Bondusphere $5 \mu \mathrm{m}$, mobile phase; methanol $/$ water $=3 / 7$ vol., flow rate; $0.7 \mathrm{ml} \mathrm{min}^{-1}$, detection; UV $210 \mathrm{~nm}$ ). A chart of the typical chromatogram is shown in Figure 1. The highest peak corresponds to monomer. Cyclic dimer was eluted before the monomer and confirmed by comparison with standard cyclic dimer. The standard cyclic dimer was extracted from nylon 6 synthesized by hydrolytic polymerization and purified and identified by nuclear magnetic resonance spectroscopy and mass spectroscopy

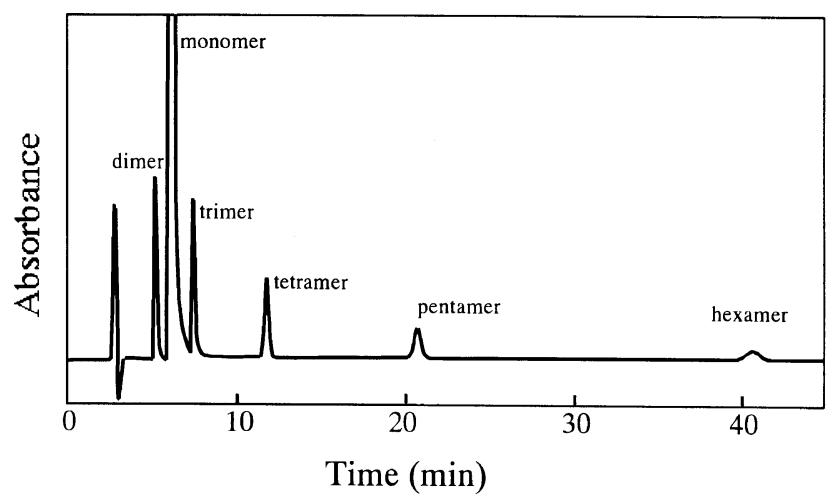

Figure 1. HPLC chromatogram of cyclic oligomers and $\varepsilon$-caprolactam. 


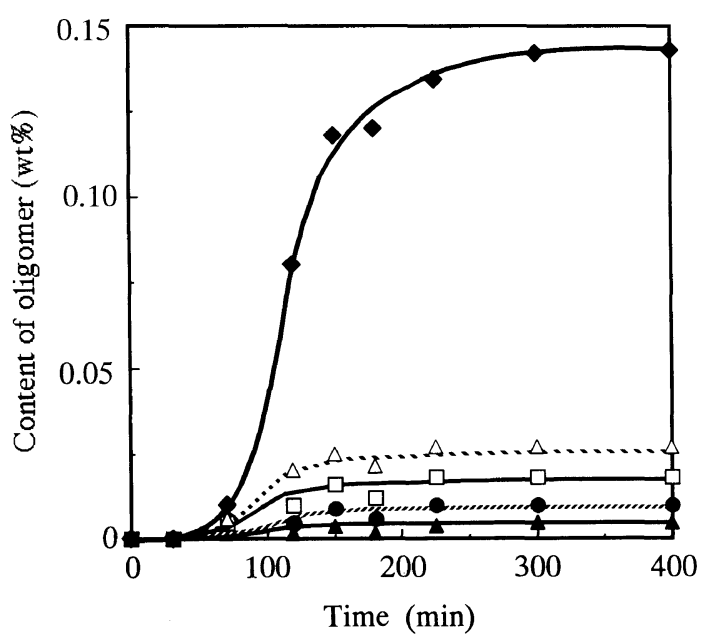

Figure 2. Relation between the content of cyclic oligomers in the polymer and polymerization time. $\diamond$, dimer; $\triangle$, trimer; $\square$, tetramer; $\boldsymbol{0}$, pentamer; $\boldsymbol{\Delta}$, hexamer. The polymerization was carried out with $0.1 \mathrm{~mol} \%$ catalyst $(\mathrm{EtMgBr})$ and $0.1 \mathrm{~mol} \%$ chain initiator (Ac-CL).

analysis. The concentration of cyclic dimer was found using the standard cyclic dimer. Cyclic trimer, tetramer, pentamer and hexamer were eluted in the order and confirmed using the standard cyclic trimer, tetramer, pentamer and hexamer, respectively. The standard cyclic trimer, tetramer, pentamer and hexamer were extracted and identified in the same way as described for cyclic dimer. ${ }^{25}$ Completeness of the extraction of the cyclic oligomers from the polymer was confirmed by dissolution of the residual polymer in formic acid, precipitation with methanol, evaporation of the solvents, retaking with water and HPLC analysis.

\section{RESULTS}

\section{Influence of Chain Initiator Concentration on Cyclic Oligomer Content}

Figure 2 shows the relationship between the content of by-produced cyclic oligomer in the polymer and polymerization time. The concentrations of the catalyst and chain initiator were both $0.1 \mathrm{~mol} \%$. The cyclic oligomers were formed very slowly up to $70 \mathrm{~min}$ and with acceleration after $100 \mathrm{~min}$. The content of cyclic dimer increased remarkably faster than that of cyclic trimer, tetramer, pentamer or hexamer. The final content of cyclic dimer was $0.14 \mathrm{wt} \%$, while that of cyclic trimer was only $0.025 \mathrm{wt} \%$. The monomer conversion was 20 $\mathrm{wt} \%$ at $22 \mathrm{~min}$, and $98.7 \mathrm{wt} \%$ at $150 \mathrm{~min}$ (ceiling value of the conversion). The contents of cyclic trimer, tetramer, pentamer and hexamer reached ceiling values around $200 \mathrm{~min}$. The content of cyclic dimer reached the ceiling value around $300 \mathrm{~min}$. Such difference in the formation behavior between cyclic dimer and the other oligomers suggests the possibility of different formation mechanisms.

Figure 3 shows the relationship between the content of by-produced cyclic oligomer in the polymer and polymerization time for $0.1 \mathrm{~mol} \%$ catalyst and 0.03 $\mathrm{mol} \%$ chain initiator. The cyclic oligomers were formed after $120 \mathrm{~min}$. The final content of cyclic dimer was $0.07 \mathrm{wt} \%$ around $500 \mathrm{~min}$ (ceiling value), this reaction time being longer than that required for reaching the

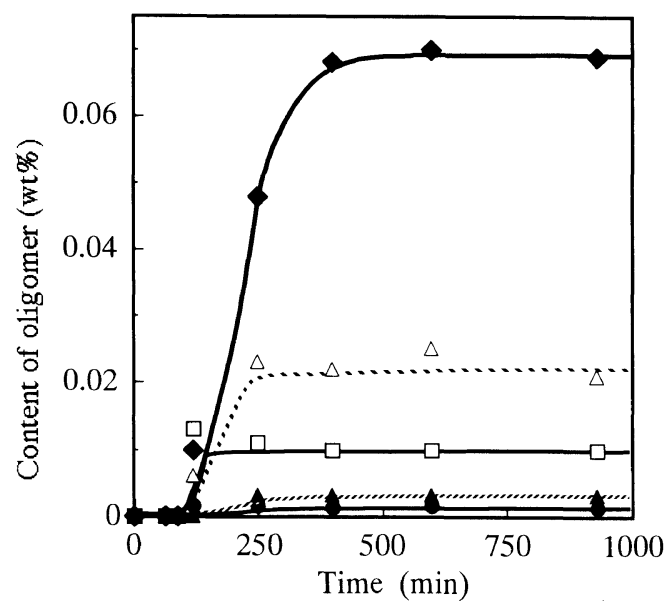

Figure 3. Relation between the content of cyclic oligomers in the polymer and polymerization time. $\diamond$, dimer; $\triangle$, trimer; $\square$, tetramer; $\boldsymbol{0}$, pentamer; $\boldsymbol{\Delta}$, hexamer. The polymerization was carried out with $0.1 \mathrm{~mol} \%$ catalyst $(\mathrm{EtMgBr})$ and $0.03 \mathrm{~mol} \%$ chain initiator (Ac-CL).

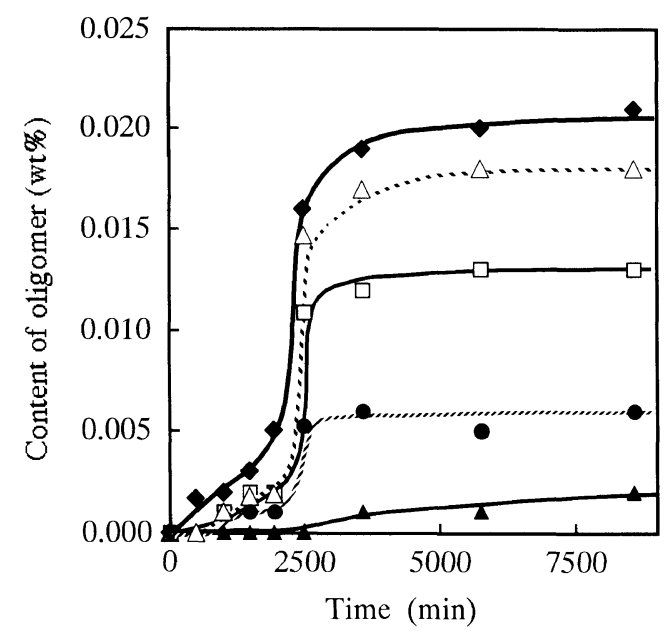

Figure 4. Relation between the content of cyclic oligomers in the polymer and polymerization time. $\diamond$, dimer; $\triangle$, trimer; $\square$, tetramer; $\boldsymbol{0}$, pentamer; $\boldsymbol{\Delta}$, hexamer. The polymerization was carried out with $0.1 \mathrm{~mol} \%$ catalyst (EtMgBr) and without chain initiator.

ceiling values of the other cyclic oligomers, as also in Figure 2. The ceiling content of cyclic dimer was $0.07 \mathrm{wt} \%$, which was the half the value in Figure 2.

Figure 4 shows the relationship between the content of by-produced cyclic oligomer in the polymer and polymerization time at $0.1 \mathrm{~mol} \%$ of the catalyst and without chain initiator. The polymerization proceeded much slower than when using the chain initiator. The final content of cyclic dimer was only $0.02 \mathrm{wt} \%$, and the period to reach the ceiling content of cyclic dimer was above $8000 \mathrm{~min}$. Cyclic trimer was formed to $0.017 \mathrm{wt} \%$, the same as that in Figures 2 and 3.

Interestingly, the rate of formation and final content (ceiling value) of cyclic dimer are strongly influenced by the presence of the chain initiator, but not those of other cyclic oligomers. To study the influence of the concentration of chain initiator on cyclic oligomer formation, the ceiling contents of the cyclic oligomers were measured by changing the concentration of chain initiator. The concentration of the catalyst was $0.1 \mathrm{~mol} \%$. The results are shown in Figure 5. The contents of the 
cyclic oligomers increased with the concentration of the chain initiator. The increments of the cyclic trimer, tetramer, pentamer and hexamer contents were small. Increase in cyclic dimer content was linear and steep in the low chain initiator concentration region and constant

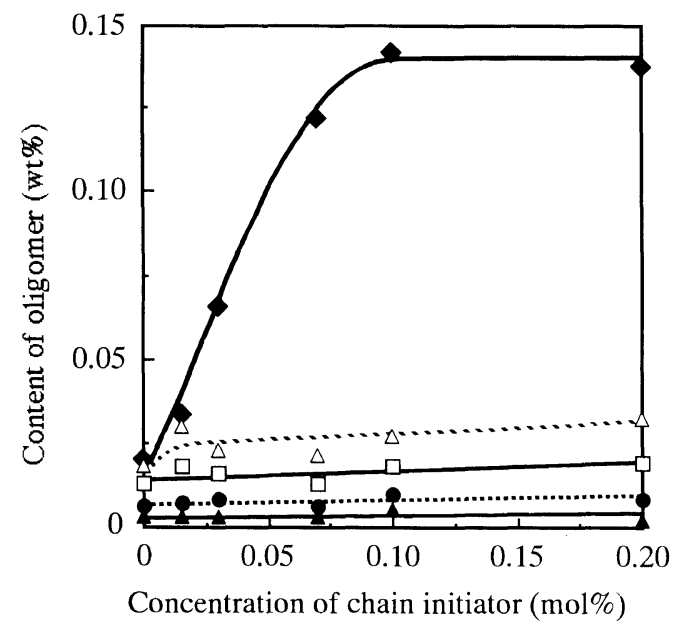

Figure 5. Influence of the concentration of chain initiator on the content of cyclic oligomers $(\diamond$, dimer; $\triangle$, trimer; $\square$, tetramer; pentamer; $\boldsymbol{\Delta}$, hexamer) in the polymer. The polymerization was carried out with $0.1 \mathrm{~mol} \%$ of catalyst $(\mathrm{EtMgBr})$.

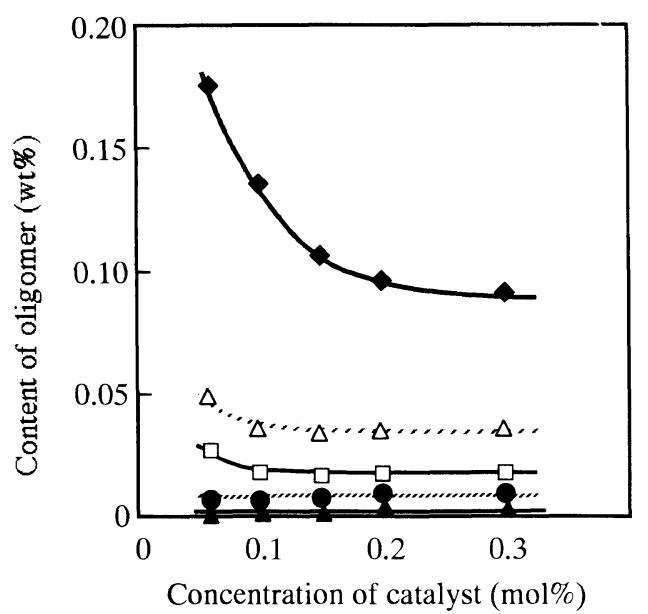

Figure 6. Influence of the concentration of catalyst on the content of cyclic oligomers $(\diamond$, dimer; $\triangle$, trimer; $\square$, tetramer; $\bullet$, pentamer; $\boldsymbol{\Delta}$, hexamer) in the polymer. The polymerization was carried out with $0.1 \mathrm{~mol} \%$ of chain initiator (Ac-CL). above $0.1 \mathrm{~mol} \%$ of the chain initiator. This suggests that the mechanisms for the formation of cyclic dimer and other cyclic oligomers differ.

\section{Influence of Catalyst Concentration on Cyclic Oligomer Content}

To investigate the influence of the catalyst concentration on cyclic oligomer formation, the ceiling contents of cyclic oligomers were measured as a function of the concentration of catalyst. The concentration of the chain initiator was $0.1 \mathrm{~mol} \%$. The results are shown in Figure 6 . The cyclic oligomer contents decreased with increase in catalyst concentration, and became constant above $0.2 \mathrm{~mol} \%$ of the catalyst. Change in cyclic dimer content was large. This suggests that there is a factor of the cyclic dimer formation, which is influenced by the catalyst concentration.

\section{DISCUSSION}

The content of each cyclic oligomer is shown in Table I, in comparison with the data published for anionic polymerization, ${ }^{21,26}$ and hydrolytic polymerization. ${ }^{26}$ The content of each cyclic oligomer in this study is 3 to 10 times smaller than that of the other studies. However, this is by no means due to any incompleteness of extraction in our cyclic oligomers analysis. We confirmed the validity of our extraction method by treating the remaining polymer aliquot, see EXPERIMENTAL. We consider that the main reason of smallness of our values is the low concentrations of chain end groups in our polymers $\left(M_{w}>10^{5}\right)$, and weak basicity of the catalyst $(\mathrm{EtMgBr})$ and extremely low water content $(0.013$ $\mathrm{mol} \%$ ). The ratios of cyclic oligomer to cyclic dimer content are also shown in Table I. Each ratio of the by-produced cyclic oligomers in the polymer synthesized with $0.1 \mathrm{~mol} \%$ catalyst and $0.1 \mathrm{~mol} \%$ chain initiator in this study is close to those in the polymer synthesized by the anionic polymerization by Biagini et al. ${ }^{21}$ Each ratio of the by-produced cyclic oligomers in the polymer synthesized without chain initiator in this study is close to those in the polymer synthesized by the hydrolytic polymerization. ${ }^{26}$ The ratio of each cyclic oligomer in the polymer synthesized with $0.03 \mathrm{~mol} \%$ of the chain initiator is intermediate between those with $0.1 \mathrm{~mol} \%$ of the chain initiator and without the chain initiator.

Table I. Content of cyclic oligomers in nylon 6

\begin{tabular}{|c|c|c|c|c|c|c|}
\hline \multirow{5}{*}{$\begin{array}{l}\text { Concentration of catalyst } / \mathrm{mol} \% \\
\text { Concentration of chain initiator } / \mathrm{mol} \% \\
\text { Polymerization temperature } /{ }^{\circ} \mathrm{C} \\
\text { Polymerization time } / \mathrm{min}\end{array}$} & \multicolumn{3}{|c|}{ This study } & \multicolumn{2}{|c|}{ Anionic polymerization ${ }^{21,26}$} & \multirow[t]{2}{*}{$\begin{array}{c}\text { Hydrolytic } \\
\text { polymerization }^{26}\end{array}$} \\
\hline & 0.1 & 0.1 & 0.1 & 0.3 & \multirow{4}{*}{$\frac{180}{-}$} & \\
\hline & 0.1 & 0.03 & 0 & 0.3 & & \multirow{3}{*}{$\begin{array}{c}250 \\
-\end{array}$} \\
\hline & 150 & 150 & 150 & 155 & & \\
\hline & 400 & 930 & 8000 & - & & \\
\hline & wt $\%$ (ratio) & wt $\%$ (ratio) & wt $\%$ (ratio) & wt $\%$ (ratio) & wt $\%$ (ratio) & wt $\%$ (ratio) \\
\hline Monomer & 1.090 & 1.082 & 2.610 & 3.04 & 1.92 & 7.80 \\
\hline Dimer & $0.142(1.00)$ & $0.066(1.00)$ & $0.021(1.00)$ & $1.02(1.00)$ & $0.29(1.00)$ & $1.13(1.00)$ \\
\hline Trimer & $0.027(0.19)$ & $0.023(0.35)$ & $0.018(0.86)$ & $0.18(0.18)$ & $0.20(0.69)$ & $0.78(0.69)$ \\
\hline Tetramer & $0.018(0.13)$ & $0.016(0.24)$ & $0.013(0.62)$ & $0.07(0.07)$ & $0.14(0.48)$ & $0.59(0.52)$ \\
\hline Pentamer & $0.010(0.07)$ & $0.008(0.12)$ & $0.006(0.29)$ & $0.04(0.04)$ & $0.11(0.38)$ & $0.45(0.40)$ \\
\hline Hexamer & $0.005(0.04)$ & $0.003(0.05)$ & $0.002(0.10)$ & $0.01(0.01)$ & $0.07(0.24)$ & $0.34(0.30)$ \\
\hline
\end{tabular}


Table II. Reactions of cyclic oligomers in the anionic polymerization of $\varepsilon$-caprolactam
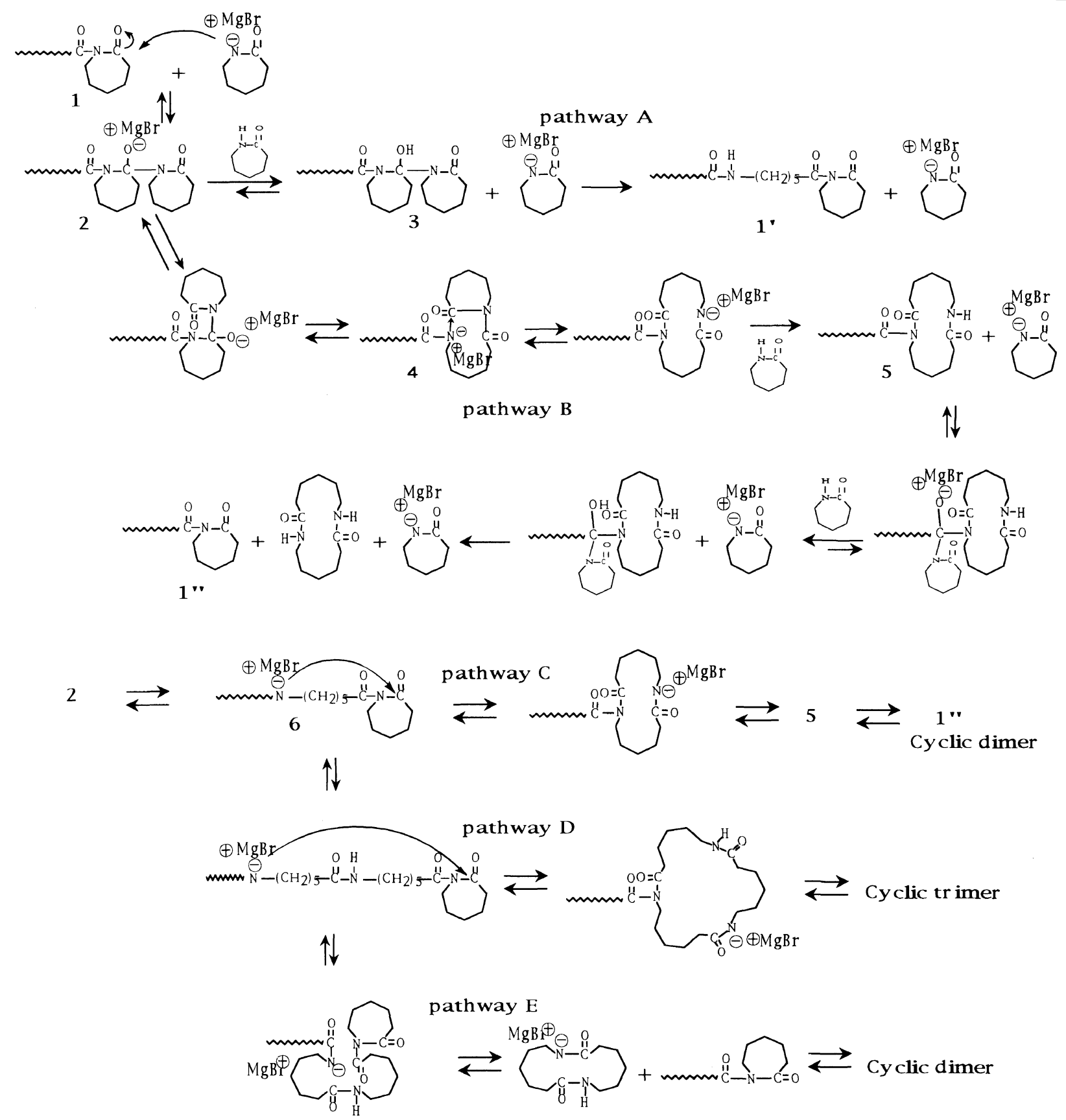

The formation of the cyclic oligomers in nylon 6 synthesized by the hydrolytic polymerization was well studied. The equilibrium of the formation of cyclic oligomers is established relatively rapidly and the experimental equilibrium contents are in accord with theoretical values. ${ }^{23,25,26}$ Cyclic oligomers in nylon 6 synthesized by hydrolytic polymerization are mainly formed by back-biting of the amino terminal group, in which an amino end-group attacks the internal amide group to form cyclic oligomers. ${ }^{26}$ The contents of cyclic oligomers in nylon 6 below $220^{\circ} \mathrm{C}$ were studied experimentally and theoretically by Andrews et al. at $186^{\circ} \mathrm{C}^{27}$ (nylon 6 is in solid state below $220^{\circ} \mathrm{C}$ ). Those at $150^{\circ} \mathrm{C}$ (the case in this study) have so far never been reported and possibly may be less than above $220^{\circ} \mathrm{C}$ and at $186^{\circ} \mathrm{C}$.
Because the back-biting by the amino terminal group scarcely occurs as low content of the amino terminal group (formed either by the hydrolysis of the polymer $^{25,26}$ or initiation reaction between lactam and lactam-anion, ${ }^{17}$ both of which reactions occur only to a limited extent $)$ and at this low temperature $\left(150^{\circ} \mathrm{C}\right)$, we consider that the equilibrium of cyclic oligomers formation was not reached in this study and would need much longer time. ${ }^{27}$

Since the cyclic dimer content and ratio to other cyclic oligomer content remarkably change with the catalyst and chain initiator concentrations, while the ratio of each cyclic oligomer at equilibrium is expected to change hardly at $150^{\circ} \mathrm{C}$ according to Andrews et al., ${ }^{27}$ a mechanism in which cyclic dimer is formed advantageously than the other cyclic oligomers would exist in the 
case of the anionic polymerization with chain initiator.

In Table II, pathway A represents the classical propagation mechanism of the anionic lactam polymerization. ${ }^{20}$ Under the normal conditions of the polymerization, reaction $2 \leftrightarrow 3$ (proton exchange between the reaction intermediate at the chain end and monomer molecule) is instantaneous. However, side reactions would sometimes occur in this reaction stage. Such as the amide-imide exchange reaction under ionized conditions and reaction $2 \leftrightarrow 4$, leading to the formation of $N$-acyl cyclic dimer 5 , which would next undergo cyclic end unit exchange with monomer anion, to release cyclic dimer (pathway B). Another reaction would be the attack of imide group of the growing chain end by linear amide anion instead of lactam anion. In fact, linear amide group increases with the conversion. Under the basic conditions of the polymerization, partly ionization would occur by the lack of lactam anion with which would give an equilibrium of ionization. When this ionization occurs on linear amide group in a chain, near its end group (for example, the one next to imide group), and if the chain conformation allows them to approach each other, they would react to form a loop. The resulting $N$-acyl cyclic oligomer would form a cyclic oligomer molecule (pathway $\mathrm{C}$ for cyclic dimer, $\mathrm{D}$ for cyclic trimer; higher cyclic oligomers possible). If the cyclic end unit is released by an internal monomolecular reaction, a cyclic oligomer lower by one monomer unit would be formed (pathway E). At present, we are not in possession of experimental results allowing us to favor or disfavor any particular pathway vis-a-vis the others.

These side reactions seem to occur from the outset of the polymerization in parallel with the propagation, to give a reaction mixture which contains, besides polymer and monomer, cyclic oligomers in ratios which decrease with increase in ring size. When the reaction mixture is amorphous (for example, in the melt at high temperatures, or before the phase solidification for the anionic polymerization) because all reactions involved are reversible, a re-equilibration of different species present begins to start to bring the cyclic oligomer composition with the value of the thermodynamically controlled mixture. This corresponds to the results of the last column in Table I (hydrolytic polymerization at $250^{\circ} \mathrm{C}^{26}$ ).

In the case of solid phase polymerization, the situation is quite different. The reaction mixture, for example with $0.1 \mathrm{~mol} \%$ catalyst and $0.1 \mathrm{~mol} \%$ chain initiator at $150^{\circ} \mathrm{C}$, solidifies at about $20 \mathrm{~min}$ of reaction. At this time, the conversion is of the order of $20 \%$, which means that the crystalline structure stretching hydrogen bonding between polymer chains is completely solvated, or even immersed, in $80 \%$ residual monomer. Molecules, ions and chain ends can move freely and reactions occur quasi-normally.

As the conversion increases, monomer becomes lacking. All reactions slow down, but the crystalline structure remains locally disorganized around the growing chain end. At about $120 \mathrm{~min}$, the field of the polymer finally becomes tight, and the chain end can no more reach the in-chain linear amide groups except the nearest one. Thus, only the cyclic dimer continues to increase in this phase of polymerization. At $300 \mathrm{~min}$, cyclic dimer ceases to be produced and the cyclic oligomer composition does not change any more. This corresponds to the results of the first column in Table I. The results of Biagini et al. ${ }^{21}$ are exactly of the same type.

For the anionic polymerization without chain initiator, for example with $0.1 \mathrm{~mol} \%$ catalyst at $150^{\circ} \mathrm{C}$, the situation is still the third type. The reaction mixture remains amorphous (at least macroscopically) for more than $500 \mathrm{~min}$, leaving time for re-equilibration to take place. In the following phase of polymerization, the crystalline structure continues to be poorly organized for a long period, so that cyclic oligomer formation and re-equilibration occur simultaneously. Thus, the resulting cyclic oligomer composition is that of a thermodynamically controlled mixture, as evident from the third column in Table I. Similar results are reported in the literature ${ }^{26}$ see the fifth column in Table I.

The contents of cyclic oligomers and ratio to cyclic dimer are intermediate the foregoing example, for polymerization using $0.1 \mathrm{~mol} \%$ catalyst and $0.03 \mathrm{~mol} \%$ chain initiator; see second column in Table I.

The anionic polymerization of $\varepsilon$-caprolactam is a good polymerization from the point of view that by-produced cyclic oligomers are formed very little.

\section{CONCLUSION}

To study the formation of cyclic oligomer in the anionic polymerization of $\varepsilon$-caprolactam, cyclic oligomer contents were measured at various catalyst and chain initiator concentrations. (1) Cyclic oligomer content increased with chain initiator concentration and decrease in catalyst concentration. (2) Cyclic dimer content was much higher than that of cyclic trimer, tetramer, pentamer and hexamer and was sensitively influenced by the catalyst and chain initiator concentrations in comparison with cyclic trimer, tetramer, pentamer and hexamer. (3) The mechanism by which cyclic dimer is advantageously formed at the terminal acyl-lactam group is proposed.

\section{REFERENCES}

1. J. Sebenda, "Comprehensive Chemical Kinetics," Vol. 15, C. H. Bamford and C. F. H. Tipper, Ed., Elsevier, Amsterdam,1976, Chapter 6.

2. H. Sekiguchi, "Ring-opening Polymerization," Vol. 2, K. J. Ivin and T. Saegusa, Ed., Elsevier, London, 1984, p 832.

3. J. Sebenda, "Comprehensive Polymer Science," Vol. 3, G. C. Eastmond,A. Ledurth, S. Russo, and P. Sigwalt, Ed., Pergamon Press, Oxford, 1989, Chapter 35.

4. R. Puffr, "Lactam-Based Polyamides," Vol. 1, R. Puffr and V. Kubánek, Ed., CRC Press, Boca Raton, Florida, 1991.

5. J. Karger-Kocsis and L. Kiss, J. Polym. Sci., Polym. Symp., 69, 67 (1981).

6. R. Puffr and N. Vladimirov, Makromol. Chem., 194, 1765 (1993).

7. J. Sebenda, J. Macromol. Sci., Chem., A6, 1145 (1972).

8. H. Sekiguchi and B. Coutin, J. Polym. Sci., Chem. Ed., 11, 1601(1973).

9. T. M. Frunze, V. A. Kotel'nikov, T. V. Volkova, and V. V. Kurashev, Eur. Polym. J., 17, 1079 (1981).

10. N. Mougin, C. A. Veith, R. E. Cohen, and Y. Gnanou, Macromolecules, 25, 2004 (1992).

11. E. Sittler and J. Sebenda, Collect. Czech. Chem. Commun., 33, 270 (1968)

12. E. Sittler and J. Sebenda, Collect. Czech. Chem. Commun., 33, 3182 (1968).

13. J. Stehlicek and J. Sebenda, Eur. Polym. J., 22, 769 (1986). 
14. T. M. Frunze, S. P. Davtyan, V. A. Kotel'nikov, T. V. Volkova and V. V. Kurashev, Vysokomol. Soedin., Ser. B, 23, 388 (1981).

15. V. G. Frolov, Vysokomol. Soedin., Ser. B, 25, 134 (1983).

16. J. Stehlicek and R. Puffr, Macromol. Chem, 193, 2539 (1992).

17. K. Ueda, K. Yamada, M. Nakai, T. Matsuda, M. Hosoda, and K. Tai, Polym. J., 28, 446 (1996).

18. K. Ueda, M. Nakai, M. Hosoda, and K. Tai, Polym. J., 28 , 1089 (1996)

19. K. Ueda, M. Nakai, K. Hattori, K. Yamada, and K. Tai, Kobunshi-Ronbunshu, 54, 401 (1997).

20. K. Ueda, M. Nakai, M. Hosoda, and K. Tai, Polym. J., 29, 568 (1997)

21. E. Biagini, G. Costa, S. Russo, and A. Turturro, Macromol.
Chem.,

Macromol. Symp., 6, 207 (1986)

22. M. Mutter, U. W. Suter, and P. J. Flory, J. Am. Chem. Soc., 98, 5745 (1976)

23. J. A. Semlyen, and G. R. Walker, Polymer, 10, 597 (1969).

24. В. В. Коршак, Т. М. Фрунзе, В. А. Котельнков, В. В. Курршев, Ю. А. Авакян, and Л. И. Комарова, Dokl. Akad. Nauk. SSSR, 275, 661 (1984).

25. K. Tai and T. Tagawa, J. Appl. Polym. Sci., 27, 2791 (1982).

26. H. K. Reimschuessel, J. Polym. Sci., Macromol. Rev., 12, 65 (1977).

27. J. M. Andrews, F. R. Jones, and J. A. Semlyen, Polymer, 15, 420 (1974). 